\title{
LATEST TECHNICAL DEVELOPMENTS AT THE INFRARED SPATIAL INTERFEROMETER
}

\author{
M. BESTER, C.G. DEGIACOMI*, W.C. DANCHI, L.J. GREENHILL**, \\ and C.H. TOWNES \\ Space Sciences Laboratory and Physics Department \\ University of California at Berkeley \\ Berkeley, CA 94720 USA \\ and \\ A. REISINGER and J. WEAVER \\ General Electric Aerospace, Electronics Laboratory \\ P.O. Box 4840 \\ Syracuse, NY 13221 USA
}

\begin{abstract}
Recent upgrades of the Infrared Spatial Interferometer are described. These provide improved sensitivity, precision and convenience of measurement. Analysis of phase fluctuations within the interferometer as well as in the atmosphere above indicates that popular simple atmospheric models need to be refined. Results of stellar observations are presented in another paper (cf. Danchi et al., in these Proceedings).
\end{abstract}

\section{General overview}

The Infrared Spatial Interferometer (ISI), located at Mount Wilson Observatory since 1988 , is a heterodyne interferometer operating in the $9-12 \mu \mathrm{m}$ atmospheric window. Baselines range from $4 \mathrm{~m}$ to $35 \mathrm{~m}$, with an optional extension up to 60 $\mathrm{m}$. Each of the two telescopes consists of a $2 \mathrm{~m}$ flat mirror and a $1.65 \mathrm{~m}$ parabolic mirror. During the last year the performance of the ISI was greatly improved, leading to an increase of the signal-to-noise ratio in fringe power by about an order of magnitude. The improvements comprise all-reflective front-end optics for beam shaping, larger bandwidth and higher quantum efficiency heterodyne detectors, better IF signal processing components, a fringe calibration system and more sensitive autoguiders. All four large mirrors were recoated at the Observatory's aluminizing facility in April 1992.

\section{Improvements of the detection system}

New heterodyne detectors were developed at the Electronics Laboratory at General Electric Aerospace, Syracuse, and tested at the ISI. They are n-on-p homojunction photodiodes fabricated by a $\mathrm{Hg}$-diffusion process which locally converts an initially p-doped substrate material into n-type wells by eliminating $\mathrm{Hg}$ vacancies. The process limits the junction depth to within $2-3 \mu \mathrm{m}$ from the top surface of the wafer. This maximizes the contribution to heterodyne detection of the highly mobile minority electrons photo-generated on the p-side of the junction, and enhances

* Present address: Spectrospin, Zurich, Switzerland.

** Present address: Harvard Smithsonian Center for Astrophysics, Cambridge, Massachusetts. 
high-frequency response. The bond pads have been reduced to their smallest practical size in order to minimize their capacitance. The IF bandwidth of our detection system is about $2000 \mathrm{MHz}$, and the quantum efficiency $\eta_{E H}$ obtained is 0.32 .

\section{Upgrade of the computer system}

The ISI computer control system was completely redesigned. The local network consists of a central UNIX host (a Sun $3 / 80$ workstation) and dedicated 68030 based VMEbus computers, one for each telescope, running VxWorks as their realtime operating system. A third VMEbus computer controls the delay line, lobe rotator, data acquisition and other system functions. Time is distributed centrally using the IRIG-B time code. This system architecture allows other workstations and real-time computers to be added in case the interferometer is expanded to more than two elements. The user interface, written in the X Window System environment, is aimed towards user friendliness and a high degree of automation of observations. Recent connection of the ISI to the Internet will eventually make remote observing possible.

\section{Analysis of atmospheric fluctuations}

Interferometric data taken with the ISI have been analyzed both to obtain information on stars and to examine atmospheric pathlength fluctuations. The latter are briefly discussed here. We find that fluctuations in pathlength through the entire atmosphere at $11 \mu \mathrm{m}$ as well as fluctuations near the ground show substantial deviations from the commonly used Kolmogorov-Taylor model. During excellent seeing conditions the outer scale of turbulence is often in the range $5-20 \mathrm{~m}$. Generally the results indicate that large-aperture telescopes and long baseline interferometers, particularly at IR wavelengths, will often perform better than is expected on the basis of the Kolmogorov-Taylor model.

Analysis of ISI data shows that under certain conditions the statistical distribution of atmospheric phase, fluctuations can be very asymmetrical. Rather often spikes appear particularly in those data sets that monitor fluctuations within the telescope optics, near the ground, representing mostly changes of air density toward a smaller index of refraction. The presence of such spikes could partially account for discrepancies in slopes between structure functions and power spectra of phase fluctuations as observed with the ISI. Samples of both phase fluctuations as a function of time and their statistical distributions are shown in Figures 1 and 2.

With the increased sensitivity of the infrared detection system a number of interferometric observations were obtained during the summer and fall of 1992 and analyzed with respect to atmospheric fluctuations. The trends observed in this enlarged data set for the slopes of structure functions, Allan variances and power spectra largely agree with those published earlier (Bester et al., 1992). The slopes are generally shallower than expected from the commonly used Kolmogorov-Taylor model, which is advantageous for the development of adaptive optics systems, i.e. the number of wavefront correcting elements employed, and for the design of longbaseline interferometers. 


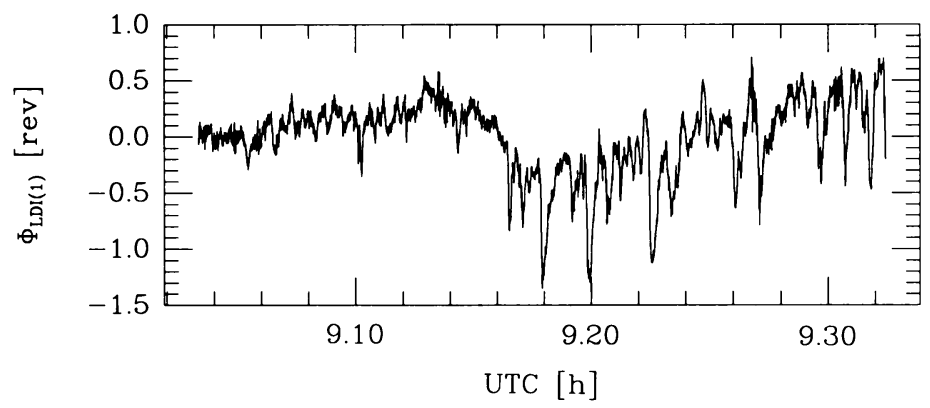

Fig. 1. Phase fluctuations measured with laser distance interferometers (LDI) on a $11 \mathrm{~m}$ long path within the telescope optics near the ground as a function of time. Units on the vertical scale are cycles at $11.15 \mu \mathrm{m}$. Bursts are clearly visible, causing the infrared phase to rotate by a full cycle.

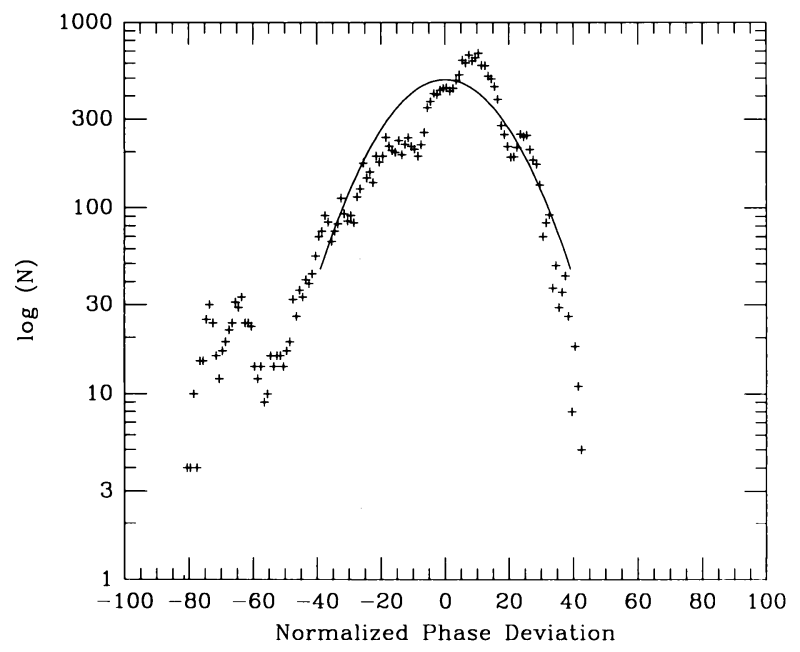

Fig. 2. Statistical distribution of the phase fluctuations in Figure 1. The bursts cause the asymmetry appearing mainly on the left side. The solid line represents a Gaussian fit to the data points.

\section{Acknowledgements}

This work was mainly supported by the United States Office of the Chief of Naval Research grant N00014-89-J-1583 and a grant from the National Science Foundation. Discussions with Robert N. Treuhaft have been very beneficial to the analysis of atmospheric fluctuations.

\section{References}

Bester, M., Danchi, W.C., Degiacomi, C.G., Greenhill, L.J., and Townes, C.H.: 1992, Astrophys. J. 392, 357 


\section{Discussion:}

Quirrenbach:

How large is the improvement in sensitivity that you expect from using delay lines? Bester:

The original estimate was a factor of 100 , based on $25 \%$ quantum efficiency of the heterodyne detectors. But since we are now using detectors with $50 \%$ quantum efficiency, the improvement is estimated to be a factor of 50 .

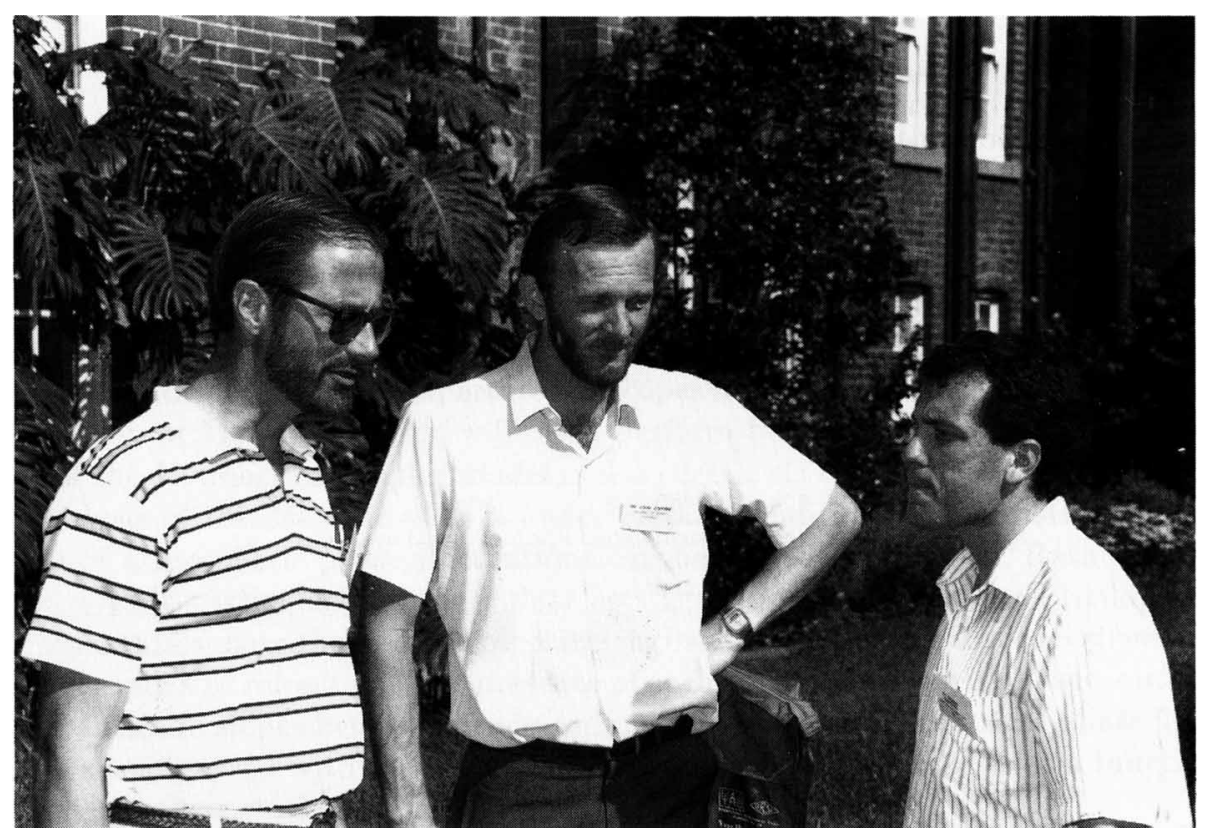

